\title{
Drug use, sexual risk behaviour and sexually transmitted infections among swingers: a cross-sectional study in The Netherlands
}

\author{
Laura W L Spauwen, ${ }^{1}$ Anne-Marie Niekamp, ${ }^{1,2}$ Christian J P A Hoebe, $^{1,2}$ \\ Nicole H T M Dukers-Muijrers ${ }^{1,2}$
}

- Additional material is published online only. To view please visit the journal online (http://dx.doi.org/10.1136/ sextrans-2014-051626).

${ }^{1}$ Department of Sexual Health, Infectious Diseases and Environmental Health, South Limburg Public Health Service, Geleen, The Netherlands 2Department of Medical Microbiology, School of Public Health and Primary Care (CAPHRI), Maastricht University Medical Center (MUMC+), Maastricht, The Netherlands

\section{Correspondence to}

L W L Spauwen, Department of Sexual Health, Infectious

Diseases and Environmental Health, South Limburg Public Health Service, PO Box 2022, 6160 HA Geleen, The Netherlands: laura.spauwen@ggdzl.nl

Received 4 April 2014 Revised 22 July 2014 Accepted 13 August 2014 Published Online First 23 October 2014

CrossMark

To cite: Spauwen LWL, Niekamp A-M, Hoebe CJPA, et al. Sex Transm Infect 2015:91:31-36.

\section{ABSTRACT}

Objectives Recreational drug use has been found to be associated with high-risk sexual behaviour and with sexually transmitted infections (STI). This study is the first to assess the prevalence of drug use among swingers (heterosexuals who, as a couple, practise mate swapping or group sex, and/or visit sex clubs for couples), and its association with high-risk sexual behaviour and STI. Methods We recruited individuals who self-identified as swingers and visited our STI clinic (from 2009 to 2012, South Limburg, The Netherlands). Participants ( $n=289$; median age 45 years; $49 \%$ female) filled in a self-administered questionnaire on their sexual and drug use behaviour while swinging, over the preceding 6 months. We assessed associations between sexual behaviour, drug use and STI diagnoses (Chlamydia trachomatis (CT), Neisseria gonorrhoeae (NG), syphilis, HIV and hepatitis B) using logistic regression analyses. Results Overall, the prevalence of CT and/or NG was $13 \%$. No other STIs were observed. Seventy-nine percent of swingers reported recreational drug use (including alcohol and use of erectile dysfunction drugs); $46 \%$ of them reported multiple drug use. Recreational drug use excluding alcohol and erectile dysfunction drugs (reported by $48 \%$ ) was associated with high-risk behaviours in men and women. Drug use was independently associated with STI in female swingers, especially those who practice group sex. Conclusions High rates of multiple drug use, as well as risky sexual behaviour and STIs among swingers, warrant paying more attention to this key population in prevention and care, as they are a risk group that is generally underrecognised and underserved in care.

\section{BACKGROUND}

Recreational drug use is associated with high-risk sexual behaviour or sexually transmitted infections (STI). ${ }^{1-5}$ Previous studies on the association between drug use and STI focused on women and on men who have sex with men (MSM), but no such data are available for swingers (heterosexuals who, as a couple, practise mate swapping or group sex, and/or visit sex clubs for couples). Swingers have recently been classified as an emerging high-risk group for STI. ${ }^{6}$ High-risk sexual behaviours, such as having multiple sex partners, group sex and a high rate of unprotected sex are common among swingers. ${ }^{6-8}$ By definition, swingers are also involved in concurrent sex, as they have overlapping partnerships over a fixed period of time. ${ }^{9}$

Participants in a pilot study among visitors to a single swingers club in Canada reported that alcohol consumption was limited. Drug use was neither reported nor observed. ${ }^{10}$ However, respondents did report high numbers engaging in unprotected sex and rarely accessing STI health services.

An earlier pilot study in The Netherlands found a higher prevalence of drug use (70\%) among a sample of 57 swinger couples. ${ }^{8}$ The prevalence of drug use was lower (55\%) among swingers who visit sex clubs. The type of drugs used was not studied. The scarce data available about swingers indicates that drug use may not be uncommon, yet we lack knowledge about the prevalence, types, combinations and frequency of drug use among swingers.

Swingers are at high risk for STI and engage in risky sex with many (concurrent) partners, yet they are generally under-recognised in healthcare. They can play a key role in the transmission of infections, both within their own network and to other populations. We hypothesised that drug use by swingers during sex is associated with risky sex and STIs. This study assessed the prevalence of multiple types of drug use while swinging by female and male swingers who visited our STI clinic. We also studied the associations between drug use and STI risk behaviour and diagnosed STI. The results might inform more efficient STI control strategies and drug use intervention strategies for swingers.

\section{METHODS}

\section{Study population}

The study population included self-identified swingers visiting the STI clinic of the South Limburg Public Health Service for STI testing, representing $16 \%$ of our clinic population. Since 2007, our clinic has systematically registered whether a visitor is a swinger or not (ie, when they were part of a heterosexual couple, and as a couple, had sex with other couples and/or singles; or couples and singles who had sex with these heterosexual couples during the past year). ${ }^{6}$ From 2009 to 2012, we approached all visiting swingers $(n=1023)$ for participation in this study. This paper presents data from the 289 participants (28\% response rate) who filled in a self-administered questionnaire after giving written informed consent, and who had been active as a swinger during the preceding 6 months. Of the non-participants, 51\% were female, their median age was 42 years (IQR: 3747), and the Chlamydia trachomatis (CT)/Neisseria gonorrhoeae (NG) point-prevalence (at their first clinic consultation) was 10\%. Between 2009 and 
2012, the proportion CT/NG positive in the total clinic population was $15 \%$, with high percentages in other risk groups as well, including young people (<25 years: 17\%), MSM (20\%), male prostitutes: $38 \%$, and female prostitute (11\%).

Medical ethical approval was obtained in 2009 from the Medical Ethical Committee of Maastricht University Medical Centre+(NL23703.068.08).

\section{Laboratory data}

Our STI clinic offers free examinations and treatment for STIs to people in risk groups, including swingers. All swingers are tested for five major STIs according to standard policy. We test for CT and NG at three different anatomic locations: on firstvoid urine (men) and self-swab (oropharyngeal in women and men, vaginal in women, and anorectal in women and men). We do this using commercially available nucleic acid amplification tests (NAAT, SDA, Becton Dickinson ProbeTec ET system, Maryland, USA or PCR, Roche Cobas Amplicor, California, USA; positive NG tests were confirmed by an inhouse PCR). Serum from venous blood punction is tested for Treponema pallidum (syphilis), HIV (anti-HIV (1/2), Axsym; Abbott, Chicago, USA); reactive samples are confirmed by western blot (HIVblot 2.2, Genelabs Diagnostics, Sciencepark, Singapore) and tested for the hepatitis B virus (HbsAg and anti-HBc, AXSYM, Abbott, Chicago, USA), following regular procedures and according to manufacturers' guidelines. Patients comprise people with symptoms (7\%), or attending after partner notification (7\%), but include mostly people attending for routine check-up.

\section{Questionnaire}

We assessed swingers' attributes using an extensive selfadministered questionnaire. Apart from demographic variables, the questionnaire comprised several known behavioural risk factors for STI. One is group sex (ie, sex with multiple persons at the same time). This definition of group sex is narrower than that used in other recent studies addressing group sex $\left(\mathrm{eg},{ }^{11}\right)$ because by definition all swingers practice group sex. Group sex was evaluated with or without using a condom. Other variables include sadomasochism (SM), number of partners in the past 6 months, use of sex toys and use of condoms. In the questionnaire, sex was defined as physical contact between hands, mouth, anus and genitals mutually, like oral, vaginal or anal sex.

In analyses, most of the variables were dichotomised: one category indicates reporting the risky behaviour and the other category indicates 'other' (including missing information). The variable 'education' was divided into 'low education' (respondents only completed elementary school and vocational education) and 'other' (respondents did not answer the question or reported a medium or high education level).

Further, all participants were asked: 'did you use drugs while swinging in the past six months?' All participants who said 'yes' were routed to questions on the types of recreational drugs (eg, methylenedioxymethamphetamine (MDMA), gamma hydroxybutyrate (GHB), laughing gas, cannabis, alkyl nitrites (poppers), (meth)amphetamines, cocaine, ketamine (LSD), lysergic acid diethylamide, psilocybin mushrooms). Separately from questions about recreational drugs, the questionnaire asked about the use of alcohol and erectile dysfunction drugs, or any combination of these substances with drugs, while swinging.

All swingers were tested for STIs. People who were diagnosed with an STI in the 12 months preceding the questionnaire date until 2 months after the questionnaire date were recorded separately as positive (at least one test was positive) or negative (all tests were negative) for the different STIs studied.

\section{Statistical analyses}

Power was sufficient $(>0.80)$ to detect an OR of at least 4 in men or in women given a proportion of the outcome of $13 \%$ (STI), or 70\% (drug use) and 50\% exposure. When exposure was lower or higher than $50 \%$, the power to detect associations was reduced.

We hypothesised that drug use during sex is associated with more risky sex and with STI in swingers (figure 1). Differences in sociodemographic characteristics, sexual behaviour, drug use and STI were compared between male and female participants using $\chi^{2}$ tests.

Determinants for the outcomes 'recreational drug use' (without alcohol or erectile dysfunction drugs) and 'STI diagnoses' (CT and NG combined) were assessed using logistic regression analyses. Drug use was used as a dichotomous variable: drug use (excluding alcohol and erectile dysfunction drugs) and non-drug use. First, sociodemographics (eg, age, educational level) and sexual behaviour were assessed as determinants for the outcome 'recreational drug use'. Second, sociodemographics, sexual behaviour and the drug use variables were assessed as determinants for the outcome 'STI diagnoses'. Third, determinants were assessed for both outcomes in a multivariate model (using a stepwise forward procedure). Only variables with a $\mathrm{p}$ value $<0.05$ in univariate analyses were included in the multivariate models. Analyses were done separately for men and women.

\section{RESULTS}

Of the 289 participants, $49 \%$ were women and 51\% were men. The participants' median age was 45 years with an IQR of $38-$ 50 years (with a minimum of 21 years and a maximum of 65 years). On average, the male participants were slightly older than the females: the median age of men was 47 years (IQR: 39-52) and the median age of women was 42 years (IQR: 35-48). The majority of the participants had been swinging for more than 3 years; they swinged at swinger clubs and/or swinged at home parties. Half the participants had been actively swinging six times or more over the preceding 6 months (table 1).

\section{STI-related risk behaviour}

One-fourth of the male participants had had sex with male swing partners in the last 6 months (MSM). Over half the participants from both sexes had had group sex in the past 6 months and half of them did not use condoms during group sex. Nine percent reported SM play and 41\% reported use of sex toys. Half the respondents reported having six or more sex partners

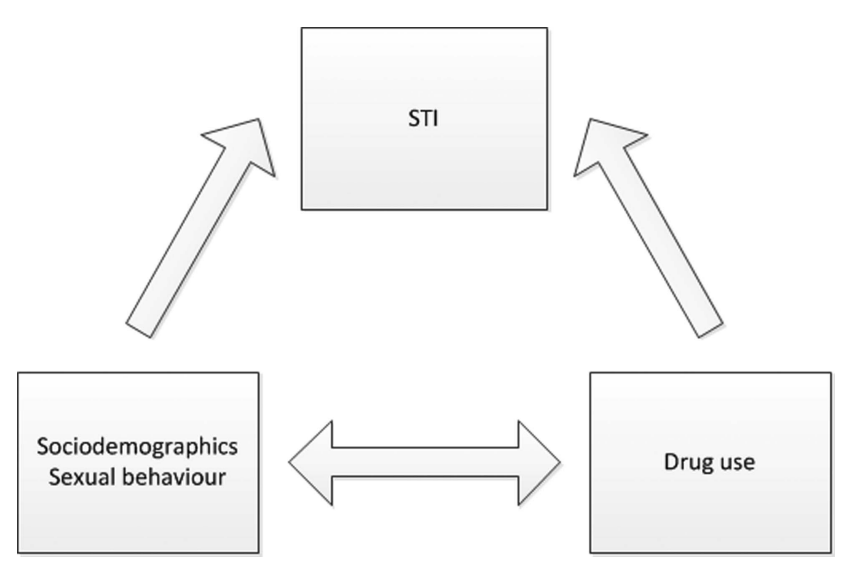

Figure 1 Association of drug use with sexual risk behaviour and STI. 
over the preceding 6 months, and had not used a condom during vaginal sex. We found no differences in sexual risk behaviour between men and women (table 1).

\section{STI prevalence}

Twenty-five participants tested positive for CT; the prevalence was $9 \%$. CT prevalence was higher in women than in men. NG was diagnosed in $5 \%$ of the participants, leading to an overall prevalence of CT and/or NG of $13 \%$, with higher positivity in women than in men. Of all $25 \mathrm{CT} / \mathrm{NG}$-positive patients, 21 were diagnosed with urogenital CT, 15 with anorectal CT, 2 with oropharyngeal CT, 3 with urogenital NG, 1 with anorectal NG and 13 with oropharyngeal NG. Over a third of the participants reported having had a previous STI (table 1).

\section{Drug use}

Nearly half of both male and female swingers reported using recreational drugs (other than alcohol or erectile dysfunction drugs) in the past 6 months while swinging. The top five drugs used were: MDMA, GHB, laughing gas, cannabis and alkyl nitrites (poppers). Nearly half the respondents reported using alcohol, and $61 \%$ of men and $8 \%$ of women reported recreational use of erectile dysfunction drugs. Multiple drug use was reported by up to $46 \%$ of all participants and was slightly higher among men. The combination of GHB and MDMA was most common (table 2).

\section{Sexual risk behaviour associated with drug use}

Overall, recreational drug use (excluding alcohol and erectile dysfunction drugs) was associated with several determinants for both men and women: swinging at home, group sex, use of toys and use of erectile dysfunction drugs were univariately associated with using recreational drugs. Additionally, low education levels, swinging for three or more years, and SM play, were univariately associated for women; for men, swinging six times or more, having sex with men, and having six or more partners, were univariately associated. Use of alcohol was inversely associated with recreational drug use for men (table 3).

In the multivariate models, swinging at home and use of toys were independent determinants for drug use in both sexes. For women, low education levels and SM play appeared to be independently associated. For men, the number of partners and no alcohol use were independent determinants for using drugs (data not shown).

\section{Sexual risk and drug use associated with STI}

In women, group sex without using a condom, use of recreational drugs, use of multiple drugs and use of erectile dysfunction drugs were univariately associated with STI diagnosis. A history of STI was univariately associated with STI for both men and women (table 3).

In the multivariate model, overall use of recreational drugs remained the only determinant associated with STI in women. Even after forcing group sex into the model, drug use remained the only independent determinant associated with STI although the risk estimate decreased $(\operatorname{adj} \mathrm{OR}=2.70$, CI 1.00 to 7.26, $\mathrm{p}<0.05$ ). It is notable that this association between drug use and STI was only present for women who participated in group sex $(O R=5.89$, CI 1.55 to 22.46) and not for women who did not participate in group sex $(\mathrm{OR}=0.74$, CI 0.13 to 4.37$)$ (the interaction between group sex and drug use was borderline significant: $\mathrm{p}=0.067$ ).

Table 1 Characteristics, sexual (risk) behaviour and STI of 289 swingers by sex

\begin{tabular}{|c|c|c|c|c|c|c|c|c|}
\hline & \multicolumn{2}{|c|}{ Men $(n=147)$} & \multicolumn{2}{|c|}{ Women (142) } & \multicolumn{2}{|c|}{ Missing data } & \multicolumn{2}{|c|}{ Total $(n=289)$} \\
\hline & $\%$ & n & $\%$ & $\mathbf{n}$ & $\%$ & $\mathbf{n}$ & $\%$ & n \\
\hline \multicolumn{9}{|l|}{ Sociodemographic } \\
\hline Age $<45$ years & 40 & 59 & 58 & 82 & 0 & 0 & 49 & $141^{*}$ \\
\hline Low education & 13 & 19 & 16 & 22 & 27 & 77 & 14 & 41 \\
\hline Swing duration 3 or more years & 74 & 108 & 67 & 95 & 6 & 16 & 70 & 203 \\
\hline Venues & & & & & 9 & 26 & & \\
\hline Ever swinged at swingers club & 77 & 113 & 77 & 109 & & & 77 & 222 \\
\hline Ever swinged at home party & 81 & 119 & 85 & 120 & & & 83 & 239 \\
\hline Swing times in past 6 months, 6 or more & 45 & 66 & 51 & 73 & 1 & 2 & 48 & 139 \\
\hline Risk behaviour in past 6 months & & & & & 9 & 25 & & \\
\hline Sex with male swing partners & 27 & 39 & 88 & 125 & & & 57 & $164^{*}$ \\
\hline Sex with female swing partners & 88 & 129 & 77 & 109 & & & 82 & 238 \\
\hline Group sex & & & & & 5 & 15 & & \\
\hline No group sex & 45 & 66 & 44 & 63 & & & 45 & 129 \\
\hline Group sex with condom & 27 & 40 & 28 & 40 & & & 28 & 80 \\
\hline Group sex without condom & 28 & 41 & 28 & 39 & & & 28 & 80 \\
\hline SM & 11 & 16 & 7 & 10 & 10 & 29 & 9 & 26 \\
\hline Six or more partners while swinging & 54 & 80 & 54 & 76 & 20 & 59 & 54 & 156 \\
\hline Use of sex toys & 42 & 61 & 40 & 57 & 14 & 41 & 41 & 118 \\
\hline No condom use vaginal sex & 57 & 84 & 56 & 80 & 22 & 63 & 57 & 164 \\
\hline STI diagnosis in past 12 months & & & & & 0 & 0 & & \\
\hline CT & 4 & 6 & 13 & 19 & & & 9 & $25^{*}$ \\
\hline NG & 4 & 6 & 6 & 8 & & & 5 & 14 \\
\hline $\mathrm{CT}$ and/or NG & 8 & 12 & 17 & 24 & & & 13 & $36^{*}$ \\
\hline STI ever (self-reported) & 38 & 56 & 41 & 58 & 1 & 4 & 39 & 114 \\
\hline
\end{tabular}


Table 2 Recreational drug use, use of alcohol and use of erectile dysfunction drugs (past 6 months) while swinging of 289 swingers by sex

\begin{tabular}{|c|c|c|c|c|c|c|}
\hline & \multicolumn{2}{|c|}{ Men $(n=147)$} & \multicolumn{2}{|c|}{ Women (142) } & \multicolumn{2}{|c|}{ Total $(n=289)$} \\
\hline & $\%$ & $\mathbf{n}$ & $\%$ & $\mathbf{n}$ & $\%$ & n \\
\hline Use of erectile dysfunction drugs & 61 & 90 & 8 & 11 & 35 & $101^{* *}$ \\
\hline Alcohol use & 43 & 63 & 49 & 70 & 46 & 133 \\
\hline \multicolumn{7}{|l|}{ Drug use while swinging } \\
\hline Drug use (excl alcohol and erectile dysfunction drugs) & 49 & 72 & 48 & 68 & 48 & 140 \\
\hline Drug use (incl alcohol and erectile dysfunction drugs) & 81 & 119 & 77 & 109 & 79 & 228 \\
\hline \multicolumn{7}{|l|}{ Top 5 drugs (other than alcohol and erectile dysfunction drugs) } \\
\hline MDMA & 43 & 63 & 41 & 58 & 42 & 121 \\
\hline GHB & 40 & 58 & 37 & 52 & 38 & 110 \\
\hline Laughing gas & 37 & 54 & 33 & 47 & 35 & 101 \\
\hline Cannabis & 31 & 46 & 27 & 38 & 29 & 84 \\
\hline Alkyl nitrites (poppers) & 17 & 25 & 15 & 21 & 16 & 46 \\
\hline \multicolumn{7}{|l|}{ Combination of drugs while swinging } \\
\hline Combination (excl alcohol and erectile dysfunction drugs) & 33 & 49 & 32 & 46 & 33 & 95 \\
\hline Combination (incl alcohol and erectile dysfunction drugs) & 52 & 77 & 49 & 56 & 46 & $133^{*}$ \\
\hline \multicolumn{7}{|l|}{ Top 3 combinations } \\
\hline GHB and MDMA & 33 & 49 & 32 & 46 & 33 & 95 \\
\hline MDMA and erectile dysfunction drugs & 31 & 46 & 4 & 5 & 18 & $51^{* *}$ \\
\hline Alcohol and erectile dysfunction drugs & 25 & 27 & 2 & 3 & 14 & $40^{* *}$ \\
\hline
\end{tabular}

\section{DISCUSSION}

To our knowledge, this is the first study to assess the prevalence of drug use among female and male swingers, and the association between drug use and STI risk. Results revealed high proportions of reported recreational drug use. Drugs were frequently used in combination during sex. Swingers are a highrisk group for STI due to their high rates of unprotected sex with multiple and concurrent partners. Together with the observed associations between drug use, risky sex and STI in female swingers, this warrants more attention for this high-risk group. Sexual behaviour that facilitates a rapid spread of STI, such as a high number of sexual partners, unprotected sex and group sex, was highly prevalent among swingers. Further, a substantial prevalence of STI was observed (13\% CT/NG-positive). These findings confirm that swingers are an important target population for STI control strategies, although they are still under-recognised and underserved in care. ${ }^{6}$ Swingers, at least those attending our clinic, are a generally older aged group. We confirm previous UK observations suggesting older STI clinic attendees, such as swingers, are a notable STI clinic population. ${ }^{6}{ }^{12}$ The prevalence of drug use observed among swingers in this study is very high and comparable with what is observed in MSM. ${ }^{1}$ Drugs were frequently used in combination: especially the combination of GHB and MDMA. Also, the combination of drugs like MDMA and erectile dysfunction drugs was frequently observed in men. It is known that some recreational drugs (eg, MDMA or GHB) may hamper the potential to ejaculate or maintain an erection. Since swingers are involved in lengthy sexual acts with multiple partners, male swingers use erectile dysfunction drugs to maintain an erection while using other drugs. It is notable that $8 \%$ of the women reported recreational use of erectile dysfunction drugs, for which the effects are unknown. We also found an association between drug use and swinging at home parties. It has previously been shown that drug use in clubs is relatively low, ${ }^{10}$ and that swingers who use drugs tend to frequently visit websites instead of clubs. ${ }^{8}$

We observed a notable association between recreational drug use and high-risk sexual behaviour. Relationships between drug use and STI are complex, but several other studies have observed associations between drug use and STI among other groups. $^{2}{ }^{13-15}$ Sexual risk taking has frequently been linked to use of alcohol and other substances by injecting drug users, MSM, patients seen in an STI clinic, youth, heterosexual users, drug offenders and young adults who routinely engage in nightlife. ${ }^{1-3} 16$ Drug use is associated with specific sexual behaviours: a higher number of sexual partners, concurrent partners, unprotected sex and lack of condom use, indiscriminate choice of sexual partner. 121718

This study only found an association between drug use and STI for female swingers. The absence of an association for males may be explained by a lack of statistical power to detect an association. On the other hand, it also corresponds with Heiligenberg et al, ${ }^{1}$ who found that sex-related drug use was associated with STI in women and MSM, but not in heterosexual men. It is difficult to specify direct effects of drug use on STI transmission. However, people who use more drugs are more likely to engage in risky sexual behaviours and have multiple or concurrent partners, which leads, in turn, to increased rates of STIs. $^{2}$

According to Friedman, group sex events (all swinger events according to our definition) can involve much use of different drugs and create an atmosphere that can lead to engaging in sex with more partners and in more dangerous ways than they had originally intended. ${ }^{11}$ Such sexual networks and social contexts can be a powerful source of influence on a number of risk behaviours including drug use, ${ }^{2} 19$ and include many factors that facilitate STI transmission. ${ }^{10}$

In addition to sexual risk behaviour, these drug effects could lead to an increased number of sexual acts, increased duration of sexual activity and increased tissue damage, blood-blood contact and blood-semen-vaginal fluid contact. ${ }^{1}$ Drug use leads to altered mental states or loss of muscle control (eg, GHB), ${ }^{20}$ decreased sensation of pain (eg, alkyl nitrites), ${ }^{21}$ intense sexual desires, and loss of inhibitions (eg, cocaine). ${ }^{22}$ Drugs like (meth) amphetamines dry the mucosa and reduce the sensitivity of the rectal and genital areas. This can facilitate longer and rougher 
Table 3 Univariate determinants for recreational drug use and diagnose with STI among 289 swingers

\begin{tabular}{|c|c|c|c|c|c|c|c|c|c|c|c|c|}
\hline & \multicolumn{6}{|c|}{ Drug use } & \multicolumn{6}{|l|}{$\underline{\text { STI }}$} \\
\hline & $\begin{array}{l}\text { OR uni } \\
\text { variate } \\
\text { men }\end{array}$ & $95 \% \mathrm{Cl}$ & $p$ Value & $\begin{array}{l}\text { OR } \\
\text { univariate } \\
\text { women }\end{array}$ & $95 \% \mathrm{Cl}$ & $\mathrm{p}$ Value & $\begin{array}{l}\text { OR } \\
\text { univariate } \\
\text { men }\end{array}$ & $95 \% \mathrm{Cl}$ & $p$ Value & $\begin{array}{l}\text { OR uni } \\
\text { variate } \\
\text { women }\end{array}$ & $95 \% \mathrm{Cl}$ & p Value \\
\hline Age $<45$ years & 0.90 & (0.47 to 1.75$)$ & 0.762 & 1.54 & (0.79 to 3.02$)$ & 0.205 & 1.55 & (0.48 to 5.05$)$ & 0.470 & 0.56 & (0.23 to 1.36$)$ & 0.199 \\
\hline Lower education & 1.94 & (0.72 to 5.25$)$ & 0.191 & 4.60 & (1.596 to 13.29 ) & 0.005 & 1.39 & (0.28 to 6.88$)$ & 0.688 & 1.11 & (0.34 to 3.63 ) & 0.862 \\
\hline Swinging more than 3 years & 1.79 & (0.85 to 3.78$)$ & 0.128 & 2.69 & (1.29 to 5.61$)$ & 0.008 & 0.70 & (0.20 to 2.47 ) & 0.579 & 0.64 & (0.26 to 1.57$)$ & 0.330 \\
\hline Ever swinged at swinger club & 1.29 & (0.60 to 2.79 ) & 0.518 & 1.33 & (0.61 to 2.92 ) & 0.474 & 0.38 & (0.11 to 1.30 ) & 0.123 & 0.89 & (0.32 to 2.47$)$ & 0.823 \\
\hline Ever swinged at home parties & 4.57 & (1.73 to 12.07$)$ & 0.002 & 5.14 & (1.64 to 16.10$)$ & 0.005 & 0.43 & (0.12 to 1.55$)$ & 0.199 & 1.34 & (0.36 to 4.96$)$ & 0.658 \\
\hline Swinging more than 6 times $^{\wedge}$ & 2.10 & (1.09 to 4.07 ) & 0.028 & 1.01 & (0.52 to 1.94 ) & 0.989 & 1.25 & (0.38 to 4.07 ) & 0.711 & 1.40 & (0.58 to 3.40$)$ & 0.458 \\
\hline Sex with a male swing partner^ & 2.70 & (1.25 to 6.81$)$ & 0.011 & N.a. & & & 1.43 & (0.41 to 5.04$)$ & 0.579 & N.a. & & \\
\hline Having group sex without condom ${ }^{\star} \wedge$ & 4.31 & (1.87 to 9.91$)$ & 0.001 & 3.42 & (1.47 to 7.98$)$ & 0.004 & 2.15 & (0.54 to 8.54$)$ & 0.275 & 3.73 & (1.25 to 11.13$)$ & 0.018 \\
\hline $\mathrm{SM}^{\wedge}$ & 2.52 & (0.83 to 7.67 ) & 0.102 & 11.14 & (1.37 to 90.42 ) & 0.024 & 1.73 & (0.34 to 8.70$)$ & 0.507 & 1.25 & (0.25 to 6.29$)$ & 0.787 \\
\hline Six partners or more ${ }^{\wedge}$ & 2.39 & (1.23 to 4.66$)$ & 0.010 & 1.20 & (0.62 to 2.32$)$ & 0.589 & 0.39 & (0.11 to 1.35$)$ & 0.137 & 1.27 & (0.52 to 3.07$)$ & 0.605 \\
\hline Use of sex toys^ & 6.15 & (2.96 to 12.75$)$ & $<0.001$ & 6.35 & (3.01 to 13.43 ) & $<0.001$ & 1.01 & (0.30 to 3.34$)$ & 0.990 & 1.99 & (0.82 to 4.82$)$ & 0.128 \\
\hline STI ever & 1.35 & (0.69 to 2.62$)$ & 0.383 & 1.64 & (0.84 to 3.22 ) & 0.150 & 10.50 & (2.14 to 51.53 ) & 0.004 & 7.38 & (2.16 to 25.16$)$ & 0.001 \\
\hline No condom use vaginal sex & 1.27 & (0.66 to 2.44 ) & 0.475 & 0.82 & (0.42 to 1.60$)$ & 0.567 & 0.42 & (0.11 to 1.61$)$ & 0.204 & 0.91 & (0.37 to 2.21$)$ & 0.829 \\
\hline Alcohol use^^ & 0.37 & (0.19 to 0.72 ) & 0.004 & 0.67 & (0.35 to 1.30$)$ & 0.238 & 0.95 & (0.29 to 3.14$)$ & 0.931 & 1.27 & (0.52 to 3.05$)$ & 0.601 \\
\hline Erectile dysfunction drugs ${ }^{\wedge}$ & 30.31 & (10.79 to 85.10$)$ & $<0.001$ & 5.49 & (1.14 to 26.41 ) & 0.034 & 1.29 & (0.37 to 4.51$)$ & 0.687 & 4.91 & (1.36 to 17.71$)$ & 0.015 \\
\hline Drug use while swinging^ & N.a. & & & & & & 0.73 & (0.22 to 2.40 ) & 0.598 & 3.19 & (1.23 to 8.27$)$ & 0.017 \\
\hline $\begin{array}{l}\text { Combination of drugs while } \\
\text { swinging } \wedge\end{array}$ & N.a. & & & & & & 1.30 & (0.39 to 4.30$)$ & 0.667 & 2.63 & (1.07 to 6.44$)$ & 0.034 \\
\hline
\end{tabular}

*Compared with no group sex.

Group sex with condom use was not significant $\wedge$ in past 6 months.

SM, sadomasochism. 
sexual episodes and contribute to an increased likelihood of bruising and lacerations in the region and of increasing opportunities for transmission of infectious disease. ${ }^{5} 1623$

Drug-using populations are a target for interventions that address the practice of safer sex along with secondary prevention of drug use. ${ }^{3}$ By dealing with all these items properly, more tailored prevention and enhanced STI screening are likely to produce gains for both individuals and the population (reduced STI burden). ${ }^{6}$

This research has several limitations. The response rate is quite low and although there seem to be no large differences between participating and non-participating swingers in age and gender, this makes it difficult to know if these findings can be extrapolated to other studies. We also acknowledge the relatively small number of swingers in this study, which limits our statistical power and ability to detect associations. For example, the power to detect associations in males and females separately was low $(<80 \%)$ to detect an OR of less than 4 . Nevertheless, this is the largest study of swingers of which we are aware. Sexual behaviour data for this study was based on self-report, which introduces recall and social desirability bias. Further, this study data was only collected from swingers who visited an STI clinic. It is unknown whether results are generalisable to swingers outside the STI clinic and/or in other countries. It should be noted that the STI prevalence was a cumulative measure over 1 year, and may thus be somewhat higher than a single-point prevalence measure. Nevertheless, previous data showed high point-prevalences as well $(10 \%){ }^{6}$ Though we used STI data about diagnoses over the 12 months preceding the questionnaire date, the questionnaire only asked about risk behaviour in the preceding 6 months. It is possible that we missed associations because of this discrepancy, but it is not likely that behaviour in the preceding 6 months differed from behaviour in the preceding 12 months.

\section{CONCLUSION}

In view of high rates of sexual risk behaviour and drug use in swingers, and given their association with STI in female swingers, swingers can play a key role in the transmission of infections. Because swingers have a complicated sexual network, they require more tailored STI control and drug use interventions that include network factors.

Key messages

- Swingers are a generally older group that shows high rates of STI, sexual risk behaviour and drug use.

- The effects of drug use on STI among female swingers are part of a context that incorporates different elements, including social network factors.

- Swingers are an important target population for STI control strategies.

- More tailored interventions for swingers are needed; these should include social network factors like group sex.

\section{Handling editor Jackie A Cassell}

Acknowledgements We acknowledge the contributions of the staff of the STI clinic South Limburg for including study participants.
Contributors All authors were involved in the conception and design of the study. A-MN and LWLS produced the database, LWLS analysed the data and wrote the first draft of the manuscript. LWLS and NHTMD-M contributed to writing the paper; all authors were involved in the final manuscript and had full access to all the data in the study, and all can take responsibility for the integrity of the data and the accuracy of the data analysis.

Competing interests None.

Patient consent Obtained.

Ethics approval Medical Ethical Committee of Maastricht University Medical Center.

Provenance and peer review Not commissioned; externally peer reviewed.

\section{REFERENCES}

1 Heiligenberg M, Wermeling PR, van Rooijen MS, et al. Recreational drug use during sex and sexually transmitted infections among clients of a city sexually transmitted infections clinic in Amsterdam, the Netherlands. Sex Transm Dis 2012;39:518-27.

2 Flom PL, Friedman SR, Kottiri BJ, et al. Stigmatized drug use, sexual partner concurrency, and other sex risk network and behavior characteristics of 18- to 24-year-old youth in a high-risk neighborhood. Sex Transm Dis 2001;28:598-607.

3 Leigh BC, Ames SL, Stacy AW. Alcohol, drugs, and condom use among drug offenders: an event-based analysis. Drug Alcohol Depend 2008;93:38-42.

4 Amirkhanian YA, Kelly JA, Takacs J, et al. HIVISTD prevalence, risk behavior, and substance use patterns and predictors in Russian and Hungarian sociocentric social networks of men who have sex with men. AIDS Educ Prev 2009;21:266-79.

5 Shoptaw S, Reback CJ. Methamphetamine use and infectious disease-related behaviors in men who have sex with men: implications for interventions. Addiction 2007;102(Suppl 1):130-5.

6 Dukers-Muijrers NH, Niekamp AM, Brouwers EE, et al. Older and swinging; need to identify hidden and emerging risk groups at STI clinics. Sex Transm Infect 2010;86:315-17.

7 Jenks RJ. Swinging: a review of the literature. Arch Sex Behav 1998;27:507-21.

8 Niekamp AM, Mercken AG, Hoebe CJPA, et al. A sexual affiliation network of swingers, heterosexuals practicing risk behaviours that potentiate the spread of sexually transmitted infections: a two-mode approach. Social Networks 2013:35:223-36.

9 Gorbach PM, Stoner BP, Aral SO, et al. "It takes a village": understanding concurrent sexual partnerships in Seattle, Washington. Sex Transm Dis 2002;29:453-62.

10 O'Byrne P, Watts JA. Exploring sexual networks: a pilot study of swingers' sexual behaviour and health-care-seeking practices. Can J Nurs Res 2011;43:80-97.

11 Friedman SR, Mateu-Gelabert P, Sandoval M. Group sex events amongst non-gay drug users: an understudied risk environment. Int J Drug Policy 2010;22:1-8.

12 Bodley-Tickell AT, Olowokure B, Bhaduri $\mathrm{S}$, et al. Trends in sexually transmitted infections (other than HIV) in older people: analysis of data from an enhanced surveillance system. Sex Transm Infect 2008;84:312-17.

13 Ross JD, Radcliffe KW. Why do those using illicit drugs have higher rates of sexually transmitted infection? Int J STD AIDS 2006;17:247-53.

14 Vivancos R, Abubakar I, Hunter PR. Sex, drugs and sexually transmitted infections in British university students. Int J STD AIDS 2008;19:370-7.

15 Ross MW, Hwang LY, Zack C, et al. Sexual risk behaviours and STIs in drug abuse treatment populations whose drug of choice is crack cocaine. Int J STD AIDS 2002;13:769-74.

16 Corsi KF, Booth RE. HIV sex risk behaviors among heterosexual methamphetamine users: literature review from 2000 to present. Curr Drug Abuse Rev 2008;1:292-6.

17 Hoffman JA, Klein $\mathrm{H}$, Eber $\mathrm{M}$, et al. Frequency and intensity of crack use as predictors of women's involvement in HIV-related sexual risk behaviors. Drug Alcohol Depend 2000;58:227-36.

18 Griffin JA, Umstattd MR, Usdan SL. Alcohol use and high-risk sexual behavior among collegiate women: a review of research on alcohol myopia theory. J Am Coll Health 2010;58:523-32.

19 Tobin KE, Latkin CA. An examination of social network characteristics of men who have sex with men who use drugs. Sex Transm Infect 2008;84:420-4.

20 Teter CJ, Guthrie SK. A comprehensive review of MDMA and GHB: two common club drugs. Pharmacotherapy 2001;21:1486-513.

21 Kurtz SP. Post-circuit blues: motivations and consequences of crystal meth use among gay men in Miami. AIDS Behav 2005;9:63-72.

22 Atkinson JS, Williams ML, Timpson SC, et al. Multiple sexual partnerships in a sample of African-American crack smokers. AIDS Behav 2010;14:48-58.

23 Volkow ND, Wang GJ, Fowler JS, et al. Stimulant-induced enhanced sexual desire as a potential contributing factor in HIV transmission. Am J Psychiatry 2007; 164:157-60. 